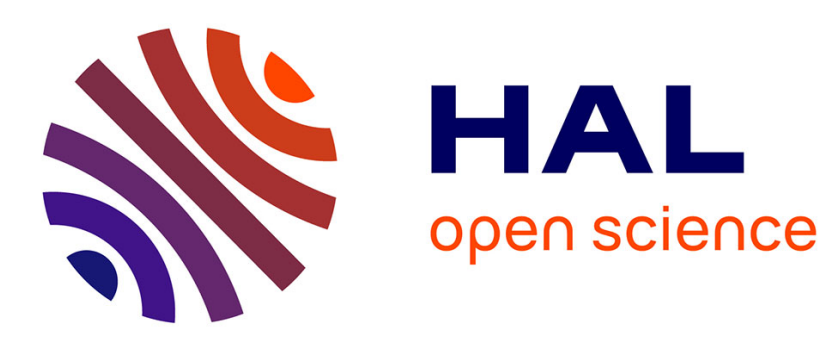

\title{
Stability of discrete-time systems with time-varying delays via a novel summation inequality
}

Alexandre Seuret, Frédéric Gouaisbaut, Emilia Fridman

\section{To cite this version:}

Alexandre Seuret, Frédéric Gouaisbaut, Emilia Fridman. Stability of discrete-time systems with timevarying delays via a novel summation inequality. IEEE Transactions on Automatic Control, 2015, 60 (10), pp.2740 - 2745. 10.1109/TAC.2015.2398885 . hal-01109796

\section{HAL Id: hal-01109796 \\ https://hal.science/hal-01109796}

Submitted on 27 Jan 2015

HAL is a multi-disciplinary open access archive for the deposit and dissemination of scientific research documents, whether they are published or not. The documents may come from teaching and research institutions in France or abroad, or from public or private research centers.
L'archive ouverte pluridisciplinaire HAL, est destinée au dépôt et à la diffusion de documents scientifiques de niveau recherche, publiés ou non, émanant des établissements d'enseignement et de recherche français ou étrangers, des laboratoires publics ou privés. 


\title{
Stability of discrete-time systems with time-varying delays via a novel summation inequality
}

\author{
Alexandre Seuret, Frédéric Gouaisbaut and Emilia Fridman
}

\begin{abstract}
This paper is concerned with the stability analysis of discrete linear systems with time-varying delays. The novelty of the paper comes from the consideration of a new inequality which is less conservative than the celebrated Jensen inequality employed in the context of discretetime delay systems. This inequality is a discrete-time counterpart of the Wirtinger-based integral inequality that was recently employed for the improved analysis of continuous-tine systems with delays. However, differently from the continuous-time case, the proof of the new inequality is not based on the Wirtinger inequality. The method is also combined with an efficient representation of the improved reciprocally convex combination inequality in order to reduce the conservatism induced by the LMIs optimization setup. The effectiveness of the proposed result is illustrated by some classical examples from the literature.
\end{abstract}

Index Terms-Summation inequalities, stability analysis, discrete-timedelay systems, time-varying delay.

\section{INTRODUCTION}

The phenomenon of delays arises in many practical situations like in biology, economy or mechanical engineering (see for instance [5]) and references therein) and its introduction in the model can lead to stability problems. This is the reason why a huge number of results is devoted to the development of criteria dedicated to the stability analysis of linear time-delay systems. When the delay is time-varying, two different methodologies have been employed. In the first framework, we aim at transforming the original system into a closed loop between a nominal LTI system and a system depending on the delay. This last element is embedded into an uncertainty and the use of classical tools like Small Gain Theorem [16], IQCs [10], [9] or Quadratic Separation [1] allows to conclude on stability.

Another technique is based on the construction of LyapunovKrasovskii functionals (see for instance [3], [4]). A first method for continuous-time delay systems is based on the so-called Complete functionals, [11], where the Lyapunov-Krasovskii functional derivative is well given and then the functional is constructed, provides necessary and sufficient stability conditions for linear systems with constant delays but hardly extendable to time-varying delays. A second popular approach relies on the a priori construction of a Lyapunov-Krasovskii functional. In this case, the design of such Lyapunov functionals is still an open problem and it is generally based on a number of steps, which somehow bring some conservatism. The first step is concerned with the choice of the Lyapunov-Krasovskii functionals. Some Lyapunov functionals are based on double or triple integral/summation terms, extended state functionals ([1], [12]), or discretization of the state ([5], [18]). Naturally, this selection entails an important conservatism. Once the increment of the Lyapunov functional calculated, the second step comes from the inequalities to be used to derive a tractable numerical optimization problem (see [6], [17], [24], [26]). In this spirit, and in the continuous-time case, all the cross terms of the derivative of $V$ are bounded using some

A. Seuret and F. Gouaisbaut are with CNRS, LAAS, 7 avenue du Colonel Roche, 31077 Toulouse, France. (e-mail: aseuret, fgouaisb@laas.fr). This work was supported by ANR project LimICoS contract number 12 BS03 00501.

E. Fridman is with Tel Aviv University, Tel Aviv, Israel. (e-mail: emilia@eng.tau.ac.il). This work was partially supported by Israel Science Foundation (grant No 754/10 and 1128/14). integral inequalities like the Jensen [5], the Wirtinger [20], [23] or the Bessel inequalities [21].

The objective of the paper is to derive simple but efficient stability conditions discrete-time time-varying delay systems. More specifically, unlike many papers in the literature, we aim at studying how to reduce the conservatism of the last stage of the procedure by the development of a less conservative summation inequality. To do so, this contribution extends [20], where integral inequalities have been provided for the stability analysis of continuous-time systems with delays, in order to provide a new summation inequality and an associated stability analysis for discrete-time systems with interval time-varying delays. More precisely, by an extensive understanding of the integral inequality from [20], we provide a new summation inequality, which is less conservative than the Jensen inequality. It is also worth mentioning that the resulting integral and summation inequalities have similar interpretations. Guided by this inequality, suitable Lyapunov- Krasovskii functionals are proposed. Finally the theoretical results are tested on an academic example and on a networked control system example.

The paper is organized as follows. Section II introduces the new summation inequality and a preliminary result on reciprocally convex inequality that will be employed. Section III presents the main results of the paper on stability of nominal and uncertain systems with timevarying delays. Section IV illustrates our results with some examples extracted from the literature.

Notations: Throughout the paper, $\mathbb{Z}$ denotes the set of integers, $\mathbb{R}^{n}$ the $n$-dimensional Euclidean space with vector norm $|\cdot|, \mathbb{R}^{n \times m}$ the set of all $n \times m$ real matrices. For any symmetric matrix $P \in \mathbb{R}^{n \times n}$, the notation $P \succ 0$ (or $P \prec 0$ ) means that $P$ is positive (or negative) definite. The set $\mathbb{S}_{n}^{+}$refers to the set of symmetric positive definite matrices. For any matrices $A, B$ in $\mathbb{R}^{n \times n}$, the notation $\operatorname{diag}(A, B)$ denotes the block diagonal matrix $\left[\begin{array}{cc}A & 0 \\ 0 & B\end{array}\right]$. For any square matrix, $\operatorname{He}(A)$ stands for $A+A^{T}$.

\section{A NOVEL SUMMATION INEQUALITIES FOR DISCRETE-TIME DELAY SYSTEMS}

\section{A. Problem formulation}

Consider a linear discrete system with time-varying delay of the form:

$$
\begin{cases}x(k+1)=A x(k)+A_{d} x(k-h(k)), & \forall k \geq 0, \\ x(k)=\phi(k), & \forall k \in\left[-h_{2}, 0\right],\end{cases}
$$

where $x(k) \in \mathbb{R}^{n}$ is the state vector, $\phi$ is the initial condition and $A$, $A_{d} \in \mathbb{R}^{n \times n}$ are constant matrices. The delay $h$ is a positive integer which is assumed to be time-varying but satisfies, for some integers $h_{2} \geq h_{1} \geq 1$,

$$
h(k) \in\left[h_{1}, h_{2}\right], \quad \forall t \geq 0 .
$$

Diverse types of functionals have been provided to assess stability of such class of systems. It has been shown in [4] that one of the most relevant terms has the following form

$$
V_{Z}\left(x_{k}\right)=\sum_{j=-h+1}^{0} \sum_{i=k+j}^{k} y^{T}(i) Z y(i),
$$


where $h$ is a positive integer, $Z$ is a positive definite matrix, $k$ is an integer representing the time variable, $x_{k}$ is the sequence of the state defined by $x_{k}(i)=x(k-i)$, for all $i=-h,-h+1, \ldots, 0$ and where $y(i)=x(i)-x(i-1)$, for all integer $i$. Computing the forward difference leads to

$$
\begin{aligned}
\Delta V_{Z}\left(x_{k}\right) & :=V_{Z}\left(x_{k+1}\right)-V_{Z}\left(x_{k}\right) \\
& =h y^{T}(k+1) Z y(k+1)-\sum_{i=k-h+1}^{k} y^{T}(i) Z y(i) .
\end{aligned}
$$

The main problem related to the equation (4) arises when including this negative term in order to derive LMI conditions. Thus, the trick is to apply the Jensen inequality, which unavoidably introduces some conservatism. In this paper, we aim at presenting a new summation inequality, which encompasses the Jensen inequality, to derive less conservative stability conditions for discrete linear systems with time-varying delays. To this aim, we take advantage of the integral inequality provided in [20] to derive a new summation inequality.

\section{B. A new summation inequality}

In this section, a novel summation is provided following the idea of [20] where a new integral inequality has been developed thanks to an extensive use of one version of the Wirtinger inequalities. This lemma is recalled here.

Lemma 1: [20] For a given symmetric positive definite matrix $R \in$ $\mathbb{S}_{n}^{+}$, any differentiable function $x$ in $[-h, 0] \rightarrow \mathbb{R}^{n}$, the following inequality holds:

$$
\int_{-h}^{0} \dot{x}^{T}(\theta) R \dot{x}(\theta) \mathrm{d} \theta \geq \frac{1}{h}\left[\begin{array}{l}
\Omega_{0} \\
\Omega_{1}
\end{array}\right]^{T}\left[\begin{array}{cc}
R & 0 \\
0 & 3 R
\end{array}\right]\left[\begin{array}{l}
\Omega_{0} \\
\Omega_{1}
\end{array}\right],
$$

where

$$
\begin{aligned}
& \Omega_{0}=x(0)-x(-h), \\
& \Omega_{1}=x(0)+x(-h)-\frac{2}{h} \int_{-h}^{0} x(\theta) \mathrm{d} \theta .
\end{aligned}
$$

The adaptation of this integral inequality into the discrete-time framework does not seem to be an easy task. Therefore, we present an alternative and simpler proof, which helps us deriving a novel summation inequality. We will also show that this summation inequality encompasses the Jensen inequality. The new proof of Lemma 1 is detailed in the following.

Proof: For any differentiable function $x$ in $[-h, 0] \rightarrow \mathbb{R}^{n}$, consider the function $z$ given by

$$
z(\theta)=\dot{x}(\theta)-\frac{1}{h} \Omega_{0}-3 \frac{(h+2 \theta)}{h^{2}} \Omega_{1}, \quad \forall \theta \in[-h, 0] .
$$

Reinjecting the definition of $z$ in $\int_{-h}^{0} z^{T}(\theta) R z(\theta) \mathrm{d} \theta$ and developing the integral yields

$$
\begin{aligned}
\int_{-h}^{0} z^{T}(\theta) R z(\theta) \mathrm{d} \theta= & \int_{-h}^{0} \dot{x}^{T}(\theta) R \dot{x}(\theta) \mathrm{d} \theta \\
& +\frac{1}{h^{2}} \int_{-h}^{0} 1 \mathrm{~d} \theta \Omega_{0}^{T} R \Omega_{0} \\
& +\frac{9}{h^{4}} \int_{-h}^{0}(h+2 \theta)^{2} \mathrm{~d} \theta \Omega_{1}^{T} R \Omega_{1} \\
& -\frac{2}{h} \int_{-h}^{0} \dot{x}^{T}(\theta) \mathrm{d} \theta R \Omega_{0} \\
& -\frac{6}{h^{2}} \int_{-h}^{0}(h+2 \theta) \dot{x}^{T}(\theta) \mathrm{d} \theta R \Omega_{1} \\
& -\frac{6}{h^{3}} \int_{-h}^{0}(h+2 \theta) \mathrm{d} \theta \Omega_{0}^{T} R \Omega_{1} .
\end{aligned}
$$

Basic integral calculus and an integration by parts ensure that

$$
\begin{array}{ll}
\int_{-h}^{0} 1 \mathrm{~d} \theta & =h, \\
\int_{-h}^{0}(h+2 \theta) \mathrm{d} \theta & =0, \\
\int_{-h}^{0}(h+2 \theta)^{2} \mathrm{~d} \theta & =\frac{1}{3} h^{3}, \\
\int_{-h}^{0} \dot{x}(\theta) \mathrm{d} \theta & =\Omega_{0}, \\
\int_{-h}^{0}(h+2 \theta) \dot{x}^{T}(\theta) \mathrm{d} \theta & =2 h \Omega_{1} .
\end{array}
$$

It thus follows that

$$
\begin{aligned}
\int_{-h}^{0} z^{T}(\theta) R z(\theta) \mathrm{d} \theta= & \int_{-h}^{0} \dot{x}^{T}(\theta) R \dot{x}(\theta) \mathrm{d} \theta \\
& -\frac{1}{h}\left(\Omega_{0}^{T} R \Omega_{0}+3 \Omega_{1}^{T} R \Omega_{1}\right) .
\end{aligned}
$$

Since $R \succ 0$, the left hand side of (8) is positive definite, which concludes the proof.

The proof of Lemma 1 relies on the construction of the function $z$, the vectors $\Omega_{0}$ and $\Omega_{1}$ and the two polynomials $1,(h+2 \theta)$. In order to understand this construction, $z$ can be interpreted as the approximation error function of the continuous function $\dot{x}$ by a first order polynomial function in $\theta$. This approximation is evaluated through the norm associated with the inner product $\int_{-h}^{0} \phi(\theta) \psi(\theta) \mathrm{d} \theta$, for any continuous functions $\phi, \psi$. In light of the consideration of the inner product, the three first equations in (7) can be interpreted as the orthogonality of the polynomials 1 and $(h+2 \theta)$ and the evaluation of their norms. Finally, the two last equations of (7) show that $\Omega_{0}$ and $2 h \Omega_{1}$ are the projections of the vector $\dot{x}$ onto these two polynomials.

It follows from this discussion, that inequality (5) is already optimized in the sense of that specific inner product. Moreover it also shows that, if the function $\dot{x}$ is a polynomial of degree 1 , then the inequality becomes an equality. More details on the construction of generalized functions $z$ are fully described in [21], [22].

Hence the idea is to translate the construction of the function $z$, the construction of the two vectors and of two polynomials but in the discrete-time framework. This summarizes the contribution of the following lemma.

Lemma 2: For a given symmetric positive definite matrix $Z \in \mathbb{S}_{n}^{+}$, any sequence of discrete-time variable $x$ in $[-h, 0] \cap \mathbb{Z} \rightarrow \mathbb{R}^{n}$, where $h \geq 1$, the following inequality holds:

$$
\sum_{i=-h+1}^{0} y^{T}(i) Z y(i) \geq \frac{1}{h}\left[\begin{array}{c}
\Theta_{0} \\
\Theta_{1}
\end{array}\right]^{T}\left[\begin{array}{cc}
Z & 0 \\
0 & 3\left(\frac{h+1}{h-1}\right) Z
\end{array}\right]\left[\begin{array}{c}
\Theta_{0} \\
\Theta_{1}
\end{array}\right],
$$

where $y(i)=x(i)-x(i-1)$ and

$$
\begin{aligned}
\Theta_{0} & =x(0)-x(-h), \\
\Theta_{1} & =x(0)+x(-h)-\frac{2}{h+1} \sum_{i=-h}^{0} x(i) .
\end{aligned}
$$

Proof: The proof is split into two cases. If $h=1$, it is easy to see that $\Theta_{1}=0$ so that equation (9) holds. Assume now that $h>1$. For any sequence $x$ in $[-h, 0] \cap \mathbb{Z} \rightarrow \mathbb{R}^{n}$, define the signal $z$ given,

$$
z(i)=y(i)-\frac{1}{h} \Theta_{0}-3 \frac{(h-1+2 i)}{h(h-1)} \Theta_{1}, \quad i=-h+1, \ldots, 1,0
$$

where $\Theta_{0}$ and $\Theta_{1}$ are defined in the statements of the lemma. The new vector $z$ has been built following the ideas given in the proof of Lemma 1. Developing $\sum_{i=-h+1}^{0} z^{T}(i) Z z(i)$ using the definition of $z$, we get

$$
\begin{aligned}
& \sum_{i=-h+1}^{0} z^{T}(i) Z z(i)=\sum_{i=-h+1}^{0} y^{T}(i) Z y(i) \\
&+\frac{1}{h^{2}}\left(\sum_{i=-h+1}^{0} 1\right) \Theta_{0}^{T} Z \Theta_{0} \\
&+\frac{9}{h^{2}(h-1)^{2}}\left(\sum_{i=-h+1}^{0}(h-1+2 i)^{2}\right) \Theta_{1}^{T} Z \Theta_{1} \\
&-\frac{2}{h} \Theta_{0}^{T} Z\left(\sum_{i=-h+1}^{0} y(i)\right) \\
&-\frac{6}{h(h-1)} \Theta_{1}^{T} Z\left(\sum_{i=-h+1}^{0}(h-1+2 i) y(i)\right) \\
&+\frac{6}{h^{2}(h-1)}\left(\sum_{i=-h+1}^{0}(h-1+2 i)\right) \Theta_{0}^{T} Z \Theta_{1} .
\end{aligned}
$$


Then some elementary calculus ensure that

$$
\begin{array}{ll}
\sum_{i=-h+1}^{0} 1 & =h, \\
\sum_{i=-h+1}^{0}(h-1+2 i) & =0, \\
\sum_{i=-h+1}^{0}(h-1+2 i)^{2} & =\frac{h\left(h^{2}-1\right)}{3}, \\
\sum_{i=-h+1}^{0} y(i) & =\Theta_{0}, \\
\sum_{i=-h+1}^{0}(h-1+2 i) y(i) & =(h+1) \Theta_{1} .
\end{array}
$$

Re-injecting them into (10) yields

$$
\begin{aligned}
\sum_{i=-h+1}^{0} z^{T}(i) Z z(i)= & \sum_{i=-h+1}^{0} y^{T}(i) Z y(i) \\
& -\frac{1}{h}\left(\Theta_{0}^{T} Z \Theta_{0}-3\left(\frac{h+1}{h-1}\right) \Theta_{1}^{T} Z \Theta_{1}\right) .
\end{aligned}
$$

The proof is concluded by noting that the left-hand side of the previous equality is positive definite.

The proofs of the lemmas for continuous and discrete-time are based on the same idea and the two inequalities are closely related. The only difference relies on the term $\frac{h+1}{h-1} \geq 1$, which is consistent with the selection $\Theta_{1}=0$ when $h=1$. Moreover, the previous proof shows that this inequality becomes an equality if $h=2$.

In some application with time-varying delays, the factor $\frac{h+1}{h-1}$ might be difficult to handle. Thus the following corollary is provided in order to make disappear this factor from the inequality.

Corollary 3: For a given symmetric positive definite matrix $Z \in$ $\mathbb{S}_{n}^{+}$, any sequence of discrete-time variable $x$ in $[-h, 0] \cap \mathbb{Z} \rightarrow \mathbb{R}^{n}$, where $h \geq 1$, the following inequality holds:

$$
\sum_{i=-h+1}^{0} y^{T}(i) Z y(i) \geq \frac{1}{h}\left[\begin{array}{c}
\Theta_{0} \\
\Theta_{1}
\end{array}\right]^{T}\left[\begin{array}{cc}
Z & 0 \\
0 & 3 Z
\end{array}\right]\left[\begin{array}{c}
\Theta_{0} \\
\Theta_{1}
\end{array}\right],
$$

where $y(i), \Theta_{0}$, and $\Theta_{1}$ are defined in Lemma 2 .

Proof: For any $h>1$, the inequality $\frac{h+1}{h-1} \geq 1$ holds. Reinjecting this inequality into equation (9) leads to the result. This equation still holds when $h=1$ because $\Theta_{1}=0$. Consequenlty, the previous equality holds for all $h \geq 1$.

Remark 1: The inequality provided in Corollary 3 (and also in Lemma 2) implies

$$
\sum_{i=-h+1}^{0} y^{T}(i) Z y(i) \geq \frac{1}{h} \Theta_{0}^{T} Z \Theta_{0},
$$

which is exactly the Jensen summation inequality. Therefore, Corollary 3 (and also in Lemma 2) is less conservative than the celebrated Jensen inequality since a positive quantity is added in the right-hand side of the inequalities.

Remark 2: The right-hand sides of the inequalities provided in Lemma 1 for continuous-time and Corollary 3 for discrete-time are exactly of the same form. In both cases, the vectors $\Omega_{0}$ and $\Theta_{0}$ represent the average evolution of the variable $x$ over the delay interval while $\Omega_{1}$ and $\Theta_{1}$ are proportional to the difference between the mean value of $x$ and its average over the delay interval.

\section{Reformulated reciprocally convex combination inequality}

In order to derive efficient stability conditions, the previous results will be combined with the convex inequality from [17] which is recalled and reformulated in the following lemma.
Lemma 4: [17] Let $n, m$ be two positive integers, and two matrices $R_{1}$ in $\mathbb{S}_{n}^{+}$and $R_{2}$ in $\mathbb{S}_{m}^{+}$. The improved reciprocally convex combination guarantees that, if there exists a matrix $X$ in $\mathbb{R}^{n \times m}$ such that $\left[\begin{array}{cc}R_{1} & X \\ X^{T} & R_{2}\end{array}\right] \succeq 0$, then the following inequality holds for any scalar $\alpha$ in the interval $(0,1)$

$$
\left[\begin{array}{cc}
\frac{1}{\alpha} R_{1} & 0 \\
0 & \frac{1}{1-\alpha} R_{2}
\end{array}\right] \succeq\left[\begin{array}{cc}
R_{1} & X \\
X & R_{2}
\end{array}\right] .
$$

Proof: By Schur complement, $\left[\begin{array}{cc}R_{1} & X \\ X^{T} & R_{2}\end{array}\right] \succeq 0$ is equivalent to $R_{1}-X R_{2}^{-1} X^{T} \succ 0$. Multiplying by the positive scalar $\frac{1-\alpha}{\alpha}$ and re-employing Schur complement, we obtain:

$0 \preceq\left[\begin{array}{cc}\frac{1-\alpha}{\alpha} R_{1} & -X \\ -X & \frac{\alpha}{1-\alpha} R_{2}\end{array}\right]=\left[\begin{array}{cc}\frac{1}{\alpha} R_{1} & 0 \\ 0 & \frac{1}{\alpha-1} R_{2}\end{array}\right]-\left[\begin{array}{cc}R_{1} & X \\ X & R_{2}\end{array}\right]$,

which is equivalent to (13).

In the remainder of the paper, stability conditions for discrete-time systems with a time-varying delay are derived based on the previous summation inequality lemma.

\section{STABILITY OF DISCRETE-TIME SYSTEMS WITH TIME-VARYING DELAYS}

\section{A. Stability result}

Based on the summation inequalities, the following stability theorem is provided.

Theorem 5: Assume that there exist matrices $P$ in $\mathbb{S}_{+}^{2 n}, Q_{1}, Q_{2}$, $Z_{1}, Z_{2}$ in $\mathbb{S}_{+}^{n}$,and a matrix $X$ in $\mathbb{R}^{2 n \times 2 n}$ such that

$$
\Psi \succ 0, \quad \Phi\left(h_{1}\right) \prec 0, \quad \Phi\left(h_{2}\right) \prec 0,
$$

where

$$
\begin{aligned}
\Phi(h)= & F_{1}^{T}\left(P+\left[\begin{array}{ccc}
h_{1}^{2} Z_{1}+h_{12}^{2} Z_{2} & 0 & 0 \\
0 & 0 & 0 \\
0 & 0 & 0
\end{array}\right]\right) F_{1} \\
& -F_{2}^{T} P F_{2}+\operatorname{He}\left(\Gamma^{T}(h) P F_{12}\right)+\hat{Q}-\Pi^{T} \Psi \Pi, \\
\Psi= & {\left[\begin{array}{ccc}
\tilde{Z}_{1}\left(h_{1}\right) & 0 & 0 \\
0 & \tilde{Z}_{2} & X \\
0 & X^{T} & \tilde{Z}_{2}
\end{array}\right], } \\
\hat{Q}= & \operatorname{diag}\left(Q_{1},-Q_{1}+Q_{2}, 0,-Q_{2}, 0,0,0\right), \\
\tilde{Z}_{1}\left(h_{1}\right)= & \operatorname{diag}\left(Z_{1}, 3 \gamma\left(h_{1}\right) Z_{1}\right), \tilde{Z}_{2}=\operatorname{diag}\left(Z_{2}, 3 Z_{2}\right),
\end{aligned}
$$

and

$$
\begin{aligned}
& F_{1}=\left[\begin{array}{ccccccc}
A-I & 0 & A_{d} & 0 & 0 & 0 & 0 \\
0 & -I & 0 & 0 & I & 0 & 0 \\
0 & 0 & -I & -I & 0 & I & I
\end{array}\right] \\
& F_{2}=\left[\begin{array}{ccccccc}
0 & 0 & 0 & 0 & 0 & 0 & 0 \\
-I & 0 & 0 & 0 & I & 0 & 0 \\
0 & -I & -I & 0 & 0 & I & I
\end{array}\right] \\
& \Gamma(h)=\left[\begin{array}{ccccccc}
I & 0 & 0 & 0 & 0 & 0 & 0 \\
0 & 0 & 0 & 0 & h_{1} I & 0 & 0 \\
0 & 0 & 0 & 0 & 0 & \left(h-h_{1}\right) I & \left(h_{2}-h\right) I
\end{array}\right] \text {, } \\
& \Pi=\left[\begin{array}{llc}
M & & 0_{2 n \times 2 n} \\
0_{2 n \times n} & M & 0_{2 n \times n} \\
0_{2 n \times 2 n} & & M
\end{array}\right], \\
& M=\left[\begin{array}{ccccc}
I & -I & 0 & 0 & 0 \\
I & I & 0 & 0 & -2 I
\end{array}\right] \text {, }
\end{aligned}
$$

and where $h_{12}=h_{2}-h_{1}, F_{12}=F_{1}-F_{2}, \gamma\left(h_{1}\right)=1$ if $h_{1}=1$ and $\gamma\left(h_{1}\right)=\left(h_{1}+1\right) /\left(h_{1}-1\right)$ if $h_{1}>1$. Then system (1) is asymptotically stable for any time-varying delay $h(k) \in\left[h_{1}, h_{2}\right]$. 
Proof : Consider the Lyapunov-Krasovskii functional given by

$$
V\left(x_{k}\right)=V_{1}\left(x_{k}\right)+V_{2}\left(x_{k}\right)+V_{3}\left(x_{k}\right),
$$

where

$$
\begin{aligned}
V_{1}\left(x_{k}\right)= & {\left[\begin{array}{c}
x(k) \\
\sum_{i=k-h_{1}}^{k-1} x(i) \\
\sum_{i=k-h_{2}}^{k-h_{1}-1} x(i)
\end{array}\right]^{T} P\left[\begin{array}{c}
x(k) \\
\sum_{i=k-h_{1}}^{k-1} x(i) \\
\sum_{i=k-h_{2}}^{k-h_{1}-1} x(i)
\end{array}\right], } \\
V_{2}\left(x_{k}\right)= & \sum_{i=k-h_{1}}^{k-1} x^{T}(i) Q_{1} x(i)+\sum_{i=k-h_{2}}^{k-h_{1}-1} x^{T}(i) Q_{2} x(i), \\
V_{3}\left(x_{k}\right)= & h_{1} \sum_{i=-h_{1}+1}^{0} \sum_{j=k+i}^{k} y^{T}(j) Z_{1} y(j) \\
& +h_{12} \sum_{i=-h_{2}+1}^{h_{1}} \sum_{j=k+i}^{k} y^{T}(j) Z_{2} y(j),
\end{aligned}
$$

where $h_{12}=h_{2}-h_{1}$ and $y(i)=x(i)-x(i-1)$. Note that the proposed functionals is the exact transcription to the discrete-time case of the functional employed in [23] for continuous-time delay systems. This functional is positive definite since $P \succ 0, Q_{1} \succ 0$, $Q_{2} \succ 0, Z_{1} \succ 0$ and $Z_{2} \succ 0$. For simplicity, the delay $h(k)$ will be denoted as $h$. In the next developments, we aim at expressing an upper bound of $\Delta V\left(x_{k}\right)$ thanks to the augmented vector

$$
\zeta(k)=\left[\begin{array}{c}
x(k) \\
x\left(k-h_{1}\right) \\
x(k-h) \\
x\left(k-h_{2}\right) \\
\nu_{1}(k) \\
\nu_{2}(k) \\
\nu_{3}(k)
\end{array}\right]
$$

where

$$
\begin{aligned}
\nu_{1}(k) & =\frac{1}{h_{1}+1} \sum_{i=k-h_{1}}^{k} x(i), \\
\nu_{2}(k) & =\frac{1}{h-h_{1}+1} \sum_{i=k-h}^{k-h_{1}} x(i), \\
\nu_{3}(k) & =\frac{1}{h_{2}-h+1} \sum_{i=k-h_{2}}^{k-h} x(i) .
\end{aligned}
$$

Therefore we aim at expressing $\Delta V_{1}$ using the augmented vector $\zeta(k)$ by noting that

$$
\begin{aligned}
{\left[\begin{array}{c}
\sum_{i=k-h_{1}}^{k-1} x(i) \\
k-h_{1}-1 \\
\sum_{i=k-h_{2}}^{k-1} x(i)
\end{array}\right]=} & {\left[\begin{array}{c}
0 \\
-x(k)+\nu_{1}(k) \\
\nu_{2}(k)+\nu_{3}(k)-x\left(k-h_{1}\right)-x(k-h)
\end{array}\right] } \\
& +\left[\begin{array}{c}
x(k) \\
h_{1} \nu_{1}(k) \\
\left(h-h_{1}\right) \nu_{2}(k)+\left(h_{2}-h\right) \nu_{3}(k)
\end{array}\right] \\
= & \left(F_{2}+\Gamma(h)\right) \zeta(k),
\end{aligned}
$$

and

$$
\begin{aligned}
{\left[\begin{array}{c}
\sum_{i=k-h_{1}+1}^{x(k+1)} x(i) \\
\sum_{i=k-h_{1}+1}^{k} x(i)
\end{array}\right]=} & {\left[\begin{array}{c}
(A-I) x(k)+A_{d} x(k-h) \\
-x\left(k-h_{1}\right)+\nu_{1}(k) \\
\nu_{2}(k)+\nu_{3}(k)-x(k-h)-x\left(k-h_{2}\right)
\end{array}\right] } \\
& +\left[\begin{array}{c}
x(k) \\
h_{1} \nu_{1}(k) \\
\left(h-h_{1}\right) \nu_{2}(k)+\left(h_{2}-h\right) \nu_{3}(k)
\end{array}\right] \\
= & \left(F_{1}+\Gamma(h)\right) \zeta(k) .
\end{aligned}
$$

Note that the previous equalities still hold when $h=h_{1}$ and $h=$ $h_{2}$. It holds

$$
\begin{aligned}
\Delta V_{1}\left(x_{k}\right)= & \zeta^{T}(k)\left[\left(F_{1}+\Gamma(h)\right)^{T} P\left(F_{1}+\Gamma(h)\right)\right. \\
& \left.-\left(F_{2}+\Gamma(h)\right)^{T} P\left(F_{2}+\Gamma(h)\right)\right] \zeta(k) \\
= & \zeta^{T}(k)\left[F_{1}^{T} P F_{1}-F_{2}^{T} P F_{2}\right. \\
& \left.+\operatorname{He}\left(\Gamma^{T}(h) P\left(F_{1}-F_{2}\right)\right)\right] \zeta(k) .
\end{aligned}
$$

On the second hand, the computation of $\Delta V_{2}\left(x_{k}\right)$ and $\Delta V_{3}$ yields

$$
\begin{aligned}
\Delta V_{2}\left(x_{k}\right)= & x^{T}(k) Q_{1} x(k)-x^{T}\left(k-h_{1}\right)\left(Q_{1}-Q_{2}\right) x\left(k-h_{1}\right) \\
& -x^{T}\left(k-h_{2}\right) Q_{2} x\left(k-h_{2}\right) \\
= & \zeta^{T}(k) \tilde{Q} \zeta(k) .
\end{aligned}
$$

and

$$
\begin{aligned}
\Delta V_{3}\left(x_{k}\right)= & y^{T}(k)^{T}\left(h_{1}^{2} Z_{1}+h_{12}^{2} Z_{2}\right) y(k) \\
& -h_{1} \sum_{i=k-h_{1}+1}^{k} y^{T}(i) Z_{1} y(i) \\
& -h_{12} \sum_{i=k-h_{2}+1}^{k-h_{1}} y^{T}(i) Z_{2} y(i) .
\end{aligned}
$$

The last sum is split into two parts: a first one gathering the terms between $k-h_{1}$ and $k-h+1$ and the second between $k-h$ and $k-h_{2}+1$. We are now in the situation to apply Lemma 2 to the first term and Corollary 3 to the two last terms. Thanks to the definitions of the matrices $F_{1}$ and $\Pi$, it yields

$$
\begin{aligned}
\Delta V_{3}\left(x_{k}\right) \leq \zeta^{T}(k) & {\left[F_{1}^{T}\left[\begin{array}{ccc}
h_{1}^{2} Z_{1}+h_{12}^{2} Z_{2} & 0 & 0 \\
0 & 0 & 0 \\
0 & 0 & 0
\end{array}\right] F_{1}\right.} \\
& \left.-\Pi^{T}\left[\begin{array}{ccc}
\tilde{Z}_{1} & 0 & 0 \\
0 & \frac{h_{12}}{h-h_{1}} \tilde{Z}_{2} & 0 \\
0 & 0 & \frac{h_{12}}{h_{2}-h} \tilde{Z}_{2}
\end{array}\right] \Pi\right] \zeta(k) .
\end{aligned}
$$

This inequality is still valid when $h$ is equal to $h_{1}$ or $h_{2}$ using a similar argument as in [14]. It consists in noting that $h_{12} /(h-$ $\left.h_{1}\right)\left(x(k-h)-x\left(k-h_{1}\right)\right)^{T} Z_{2}\left(x(k-h)-x\left(k-h_{1}\right)\right)$ is zero when $h=h_{1}$. A similar argument holds when $h=h_{2}$. Finally the convexity argument of Lemma 4 ensures that, if there exists a matrix $X \in \mathbb{R}^{2 n \times 2 n}$ such that $\Psi \succ 0$, then the following upper bound of $\Delta V_{d 3}$ is obtained

$$
\begin{aligned}
\Delta V_{3}\left(x_{k}\right) \leq \zeta^{T}(k) & {\left[F_{1}^{T}\left[\begin{array}{ccc}
h_{1}^{2} Z_{1}+h_{12}^{2} Z_{2} & 0 & 0 \\
0 & 0 & 0 \\
0 & 0 & 0
\end{array}\right]\right.} \\
& \left.-\Pi^{T} \Psi \Pi\right] \zeta(k) .
\end{aligned}
$$

Re-injecting the expressions of $\Delta V_{1}$ and $\Delta V_{2}$ and the upper bound of $\Delta V_{3}$ into the expression of $\Delta V$ leads to

$$
\Delta V\left(x_{k}\right) \leq \zeta^{T}(k) \Phi(h) \zeta(k)
$$

Since the matrix $\Phi(h)$ is affine with respect to the delay $h, \Phi(h)$ is definite negative if and only if $\Phi\left(h_{1}\right)$ and $\Phi\left(h_{2}\right)$ are negative definite, which concludes the proof.

Remark 3: Theorem 5 is the discrete-time version of the result showed in [23] for continuous-time systems with interval timevarying delays.

B. Robust stability of discrete-time systems with time-varying delays

Consider an uncertain linear discrete-time delay system of the form:

$\left\{\begin{array}{l}x(k+1)=\sum_{i=0}^{M} \lambda_{i}(k)\left\{A_{i} x(k)+A_{d i} x(k-h(k))\right\}, \forall k \geq 0, \\ x(k)=\phi(k), \quad \forall k \in\left[-h_{2}, 0\right],\end{array}\right.$ 


\begin{tabular}{|c|c|c|c|c|c|c|c|}
\hline$h_{1}$ & 1 & 3 & 5 & 7 & 11 & 13 & N of variables \\
\hline \hline$[4]$ & 10 & 10 & 11 & 12 & 13 & 13 & $2.5 n^{2}+2.5 n$ \\
{$[27]$} & 12 & 13 & 14 & 15 & 17 & 19 & $9 n^{2}+3 n$ \\
{$[7]$} & 17 & 17 & 17 & 18 & 20 & 22 & $13 n^{2}+5 n$ \\
{$[25]$} & 17 & 17 & 18 & 18 & 20 & 23 & $8 n^{2}+3 n$ \\
{$[9]$} & 17 & 18 & 19 & 21 & 25 & 25 & $\frac{n^{2}}{2}\left(h_{2}+1\right)^{2}+\frac{n}{2}\left(h_{2}+2\right)$ \\
{$[13]$} & 22 & 22 & 22 & 22 & 23 & 24 & $27 n^{2}+9 n$ \\
\hline \hline Th.5 & 20 & 21 & 21 & 22 & 23 & 24 & $10.5 n^{2}+3.5 n$ \\
\hline
\end{tabular}

ADMISSIBLE UPPER BOUND $h_{2}$ FOR VARIOUS $h_{1}$ FOR THE SYSTEM DESCRIBED IN EXAMPLE (20).

where $x(k) \in \mathbb{R}^{n}$ is the state vector, $\phi$ is the initial condition and $A_{i}, A_{d i} \in \mathbb{R}^{n \times n}$ are constant and given matrices. The time-varying coefficients $\lambda_{i}$ belong to the unit simplex and satisfy

$$
\forall i=0, \ldots, M, \quad \lambda_{i}(k) \geq 0, \quad \sum_{i=0}^{M} \lambda_{i}(k)=1 .
$$

The delay function is assumed to verify the same constraints as in (2). The following theorem provides an extension of Theorem 5 to the case of uncertain systems

Theorem 6: Assume that there exist matrices $P$ in $\mathbb{S}_{+}^{2 n}, Q_{1}, Q_{2}$, $Z_{1}, Z_{2}$ in $\mathbb{S}_{+}^{n}$, and a matrix $X$ in $\mathbb{R}^{2 n \times 2 n}$ such that $\Psi \succ 0$ and

$$
\forall i=1 \ldots M, \quad \Phi_{i}\left(h_{1}\right) \prec \quad 0, \quad \Phi_{i}\left(h_{2}\right) \quad \prec \quad 0,
$$

where

$$
\begin{aligned}
\Phi_{i}(h)= & F_{1 i}^{T}\left(P+\left[\begin{array}{ccc}
h_{1}^{2} Z_{1}+h_{12}^{2} Z_{2} & 0 & 0 \\
0 & 0 & 0 \\
0 & 0 & 0
\end{array}\right]\right) F_{1 i} \\
& -F_{2}^{T} P F_{2}+\operatorname{He}\left(\Gamma^{T}(h) P F_{12 i}\right)+\hat{Q}-\Pi^{T} \Psi \Pi,
\end{aligned}
$$

where the matrices $\Psi, \Gamma(h), \Pi, \hat{Q}, \tilde{Z}_{1}, \tilde{Z}_{2}, F_{2}$ and the parameters $h_{12}, \gamma(h)$ are given in the statement of Theorem 5 and where

$$
\begin{aligned}
F_{1 i} & =\left[\begin{array}{ccccccc}
A_{i}-I & 0 & A_{d i} & 0 & 0 & 0 & 0 \\
0 & -I & 0 & 0 & I & 0 & 0 \\
0 & 0 & -I & -I & 0 & I & I
\end{array}\right], \\
F_{12 i} & =F_{1 i}-F_{2} .
\end{aligned}
$$

Then system (17) is robustly asymptotically stable for any timevarying delay $h(k) \in\left[h_{1}, h_{2}\right]$.

Proof: The proof follows straightforwardly by use of Schur complement, which ensures the convexity of the LMI condition (14) with respect to the system matrices $A$ and $A_{d}$.

\section{EXAMPLES}

\section{A. Stability example}

Consider the following discrete-time linear time-delay system (1): $x(k+1)=\left[\begin{array}{rr}0.8 & 0.0 \\ 0.05 & 0.9\end{array}\right] x(k)+\left[\begin{array}{rr}-0.1 & 0.0 \\ -0.2 & -0.1\end{array}\right] x(k-h(k))$.

A detailed comparison to various theorems from the literature is provided in Table I. It is worth mentioning that the benefits of employing the new summation inequalities from Lemma 2 and Corollary 3 are twofold. It indeed requires less decision variables than in the existing conditions [13] while leading to the same result.

\begin{tabular}{|c|c|c|c|c|c|c|c|}
\hline$T_{1}=0.1$ & $T_{2}$ & 0.11 & 0.15 & 0.2 & 0.3 & 0.5 & 0.64 \\
\hline$h_{1}=1$ & $h_{2}$ & 9 & 6 & 4 & 3 & 1 & 1 \\
\hline
\end{tabular}

ADMISSIBLE UPPER BOUND $h_{2}$ FOR VARIOUS $T_{2}$ WITH $T_{1}=0.1$ AND $h_{1}=1$ FOR SYSTEM DESCRIBED IN EXAMPLE (22).

\begin{tabular}{|c|c|c|c|c|c|}
\hline$T_{1}=0.1$ & $T_{2}$ & 0.11 & 0.15 & 0.2 & 0.202 \\
\hline$h_{1}=5$ & $h_{2}$ & 9 & 6 & 5 & 5 \\
\hline
\end{tabular}

ADMISSIBLE UPPER BOUND $h_{2}$ FOR VARIOUS $T_{2}$ WITH $T_{1}=0.1$ AND $h_{1}=5$ FOR SYSTEM DESCRIBED IN EXAMPLE (22).

\section{B. Robust stability : Application to networked control systems}

Consider the linear sampled-data system borrowed from [28] given, for all $t$ in $\left[t_{k}, t_{k+1}\right)$ by

$$
\dot{x}(t)=\left[\begin{array}{cc}
0 & 1 \\
0 & -0.1
\end{array}\right] x(t)+\left[\begin{array}{cc}
0 & 0 \\
-0.375 & -1.15
\end{array}\right] x\left(t_{k-h(k)}\right),
$$

where $\left\{t_{k}\right\}_{k \geq 0}$ represents a strictly increasing sequence of time instants for which there exist positive scalars $0<T_{1}<T_{2}$ such that

$$
T_{k}:=t_{k+1}-t_{k} \in\left[T_{1}, T_{2}\right], \quad \forall k \geq 0 .
$$

This class of examples differs notably from the problem addressed in [15], [2], where the control input is computed with $x\left(t_{k}-\tau\right)$, where $\tau$ is a positive scalar representing a continuous-time delay. Here, the sampling instants and the delayed samples employed to generate the sampled input coincide. This time-delay example has been already studied in [19] but only in the case of constant delay $h$. The discretization of the sampled-data system leads to

$$
x(k+1)=A\left(T_{k}\right) x(k)+A_{d}\left(T_{k}\right) x\left(t_{k-h(k)}\right), \quad \forall k \geq 0,
$$

where

$$
A\left(T_{k}\right):=e^{\left[\begin{array}{cc}
0 & 1 \\
0 & -0.1
\end{array}\right] T_{k}}=\left[\begin{array}{cc}
1 & 10\left(1-\lambda\left(T_{k}\right)\right) \\
0 & \lambda\left(T_{k}\right)
\end{array}\right],
$$

and

$$
\begin{aligned}
A_{d}\left(T_{k}\right)=\int_{0}^{T_{k}} e^{\left[\begin{array}{ll}
0 & 1 \\
0 & -0.1
\end{array}\right] s}\left[\begin{array}{cc}
0 & 0 \\
-0.375 & -1.15
\end{array}\right] d s \\
\quad=\left[\begin{array}{cc}
32.5\left(1-\lambda\left(T_{k}\right)\right)-3.75 T_{k} & 115\left(1-\lambda\left(T_{k}\right)\right)-11.5 T_{k} \\
3.75\left(\lambda\left(T_{k}\right)-1\right) & 11.5\left(\lambda\left(T_{k}\right)-1\right)
\end{array}\right],
\end{aligned}
$$

with the notation $\lambda\left(T_{k}\right)=e^{-T_{k} / 10}$. Using the convexity property of the exponential function, we show that

$$
\begin{aligned}
\left(T_{k}, \lambda\left(T_{k}\right)\right) \in \mathcal{C} o\{ & \left(T_{m}, \lambda\left(T_{m}\right)\right),\left(T_{M}, \lambda\left(T_{M}\right)\right), \\
& \left.\left(T_{m}, \lambda\left(T_{M}\right)\left(1+\left(T_{M}-T_{m}\right) / 10\right)\right)\right\},
\end{aligned}
$$

where the notation $\mathcal{C} O$ refers to the convex of hull the considered vertices. The vertex $\left(T_{m}, \lambda\left(T_{M}\right)\left(1+\left(T_{M}-T_{m}\right) / 10\right)\right)$ has been computed thanks to the tangent line of the exponential function $T \rightarrow$ $\lambda(T)=e^{-T / 10}$ at $T=T_{M}$. Then, a polytopic modeling of the discrete-time system can be defined and the system (22) becomes suitable for the application of Theorem 6 . The results are given in Table II where a tradeoff appears between the allowable uncertainty in the sampling period and the allowable variations in the delay function.

When the delay is constant and $h_{1}=h_{2}=1$ and $h_{1}=h_{2}=5$, an eigenvalue analysis shows that the maximal acceptable constant sampling period $T_{m}=T_{M}=T$ for which the system remains asymptotically stable is $T=0.76$ and $T=0.21$, respectively. Note that as we have mentioned earlier in the paper, the summation inequality is not conservative when $h_{1}=h_{2}=1$. Hence, the conservatism in this case comes from the approximation the uncertain system (22). This conservatism may be reduced by employing more 
advanced approximation methods provided for instance in [8]. This illustrates that the stability analysis provided in this paper is not conservative.

\section{Conclusions}

In this paper, a new summation inequality is presented and is shown to be relevant for the stability analysis of discrete-time systems with interval time-varying delays. This inequality has been derived from an appropriate interpretation of the Wirtinger-based integral inequality and is proved to be less conservative than the usual Jensen inequality. Combined with the reciprocal convexity lemma, two stability theorems have been provided, which are less conservative or less computationally demanding than the usual Lyapunov-Krasovskii approaches together with the Jensen inequality.

\section{REFERENCES}

[1] Y. Ariba, F. Gouaisbaut, and K.H. Johansson. Robust stability of timevarying delay systems: Quadratic separation approach. Asian Journal of Control, 14(5):1205-1214, 2012.

[2] M.B.G. Cloosterman, N. van de Wouw, W.P.M.H. Heemels, and H. Nijmeijer. Stability of networked control systems with uncertain timevarying delays. IEEE Trans. on Automatic Control, 54(7):1575-1580, 2009.

[3] E. Fridman and U. Shaked. Delay dependent stability and $H_{\infty}$ control: constant and time-varying delays. Int. J. of Control, 76(1):48-60, 2003.

[4] E. Fridman and U. Shaked. Stability and guaranteed cost control of uncertain discrete delay systems. Int. J. Control, 78(4):235-246, 2005.

[5] K. Gu, V.-L. Kharitonov, and J. Chen. Stability of time-delay systems. Birkhauser, 2003.

[6] Y. He, Q. G. Wang, L. Xie, and C. Lin. Further improvement of freeweighting matrices technique for systems with time-varying delay. IEEE Trans. on Automatic Control, 52(2):293-299, 2007.

[7] Y. He, M. Wu, G.-P. Liu, and J.-H. She. Output feedback stabilization for a discrete-time system with a time-varying delay. IEEE Trans. on Automatic Control, 53(10):2372-2377, 2008.

[8] W.P.M.H. Heemels, N. Van De Wouw, R.H. Gielen, M.C.F. Donkers, L. Hetel, S. Olaru, M. Lazar, J. Daafouz, and S. Niculescu. Comparison of overapproximation methods for stability analysis of networked control systems. In ACM International Conference on Hybrid Systems: Computation and Control, pages 181-190. ACM, 2010.

[9] C.-Y. Kao. On stability of discrete-time LTI systems with varying time delays. IEEE Trans. on Automatic Control, 57(5):1243-1248, 2012

[10] C.Y. Kao and A. Rantzer. Stability analysis of systems with uncertain time-varying delays. Automatica, 43(6):959 - 970, 2007.

[11] V.L. Kharitonov and A.P. Zhabko. Lyapunov-Krasovskii approach to robust stability of time delay systems. In In First IFAC/IEEE symposium on system structure and control, Praha, Czech Rep., 2001.

[12] J.H. Kim. Note on stability of linear systems with time-varying delay. Automatica, 47(9):2118-2121, 2011.

[13] O.M. Kwon, M.J. Park, J.H. Park, S.M. Lee, and E.J. Cha. Stability and stabilization for discrete-time systems with time-varying delays via augmented Lyapunov-Krasovskii functional. J. of the Franklin Institute, 350(3):521-540, 2013.

[14] J. Liu and J. Zhang. Note on stability of discrete-time time-varying delay systems. IET Control Theory and Applications, 6(2):335-339, 2012.

[15] K. Liu and E. Fridman. Wirtinger's inequality and Lyapunov-based sampled-data stabilization. Automatica, 48(1):102-108, 2012.

[16] L. Mirkin. Some remarks on the use of time-varying delay to model sample-and-hold circuits. IEEE Trans. on Automatic Control, 52(6): 1009-1112, 2007.

[17] P.G. Park, J. W. Ko, and C. Jeong. Reciprocally convex approach to stability of systems with time-varying delays. Automatica, 47(1):235238, 2011.

[18] C. Peng. Improved delay-dependent stability criteria for discrete-time systems with a new finite sum inequality. IET Control Theory and Applications, 6(3):448-453, 2012.

[19] A. Seuret, C. Briat, and F. Gouaisbaut. Stability analysis of asynchronous sampled-data systems with discrete-time constant input delay. IEEE Conference on Decision and Control (CDC'14), 2014.

[20] A. Seuret and F. Gouaisbaut. Wirtinger-based integral inequality: Application to time-delay systems. Automatica, 49(9):2860-2866, 2013.
[21] A. Seuret and F. Gouaisbaut. Complete quadratic Lyapunov functionals using Bessel-Legendre inequality. European Control Conference (ECC’14), 2014.

[22] A. Seuret and F. Gouaisbaut. Hierarchy of LMI conditions for the stability analysis of time-delay systems. submitted to Systems \& Control Letters, 2015.

[23] A. Seuret, F. Gouaisbaut, and E. Fridman. Stability of systems with fastvarying delay using improved Wirtinger's inequality. IEEE Conference on Decision and Control (CDC'13), pages 946-951, 2013.

[24] H. Shao. New delay-dependent stability criteria for systems with interval delay. Automatica, 45(3):744-749, 2009.

[25] H. Shao and Q.L. Han. New stability criterion for linear discretetime systems with interval-like time-varying delays. IEEE Trans. on Automatic Control, 56(3):619-625, 2011.

[26] J. Sun, G.P. Liu, J. Chen, and D. Rees. Improved delay-range-dependent stability criteria for linear systems with time-varying delays. Automatica, 46(2):466-470, 2010.

[27] B. Zhang, S. Xu, and Y. Zou. Improved stability criterion and its applications in delayed controller design for discrete-time systems. Automatica, 44(11):2963-2967, 2008.

[28] W. Zhang, M.S. Branicky, and S.M. Phillips. Stability of networked control systems. IEEE Control Systems Magazine, (21), 2001. 